\title{
Comparison of Different Methods for Estimating Fractal Dimension with the Method of Statistics
}

\author{
Matej Babič ${ }^{1, *}$
}

Jožef Stefan Institute

\begin{abstract}
This paper presents three methods for calculating the fractal dimension and its application in Mechanical Engineering. The microstructure of hardened specimens is very complex and we cannot describe it with classical Euclidian geometry. Thus we use a new method, fractal geometry. We present the method of box-counting, homeomorphic model and the R/S method to calculate fractal dimensions. We compare how these three different methods have an impact on the mechanical properties of robot laser hardened specimens. We use the statistical method of multiple regression to describe the percentage of black pixels of SEM images of robot laser hardened specimens. Robot laser hardening is a technology of heat treatment process. Today, technologists who operate various CNC machines only have a knowledge based on practical experience. Each technologist must consider numerous CNC machine parameters to obtain the desired results. Because this is a very time-consuming process, we used a statistical method, which allows us to obtain results more quickly. Heat treatment is known as a quicker way to access information according to the desired specifications. It was necessary to use a statistical method based on individual samples to disclose the percentage of black pixels of SEM images of robot laser hardened specimens. With this statistical method, we increase the production of the process of laser hardening, because we decrease the time of the process and increase the topographical properties of materials.
\end{abstract}

Keywords: Statistics, fractal dimension, hardening, multiple regression.

\section{Introduction}

Statistics is an important tool to handle the vast data of the present era, as statistics can interpret all the information in such a way that many conclusions can be extracted from it. It is the study of the collection, analysis, interpretation, presentation, and organisation of data. Thus, statistics can be called that body of analytical and computational methods by which the characteristics of a population are inferred through observations made in a representative sample from that population. Although the objective of statistical methods is to make the process of scientific research as efficient and productive as possible, many scientists and engineers have inadequate training in experimental design and in the proper selection of statistical analyses for experimentally acquired data. Nature is full of shapes and images, which from a distance may seem similar to each other (i.e. Self-Similarity Briefings). A well-known example of such a self-similar pattern is Sierpinski's pyramid. Similarly, we can define the field of statistics. Let us forever, 
in itself statistically similar data series. Any part of this series would have the same statistical characteristics as the entire series or any other part of this series. Of all microscopic methods, electron microscopy images give the best resolution, the most accurate information of the distribution of crystals in the building, the best morphology of the various structural types and the best structural surface. In fractal geometry [1], the fractal dimension, D [2-4] is a statistical quantity that gives an indication of how completely a fractal appears to fill space as one zooms down to finer and finer scales. A fractal surface consists of many complicated zigzag planes. Recently, the concept of the fractal dimension has been applied to the analysis of these surfaces. Figure 1 presents the statistical self-affinity fractal structure of SEM pictures of robot laser hardened specimens.
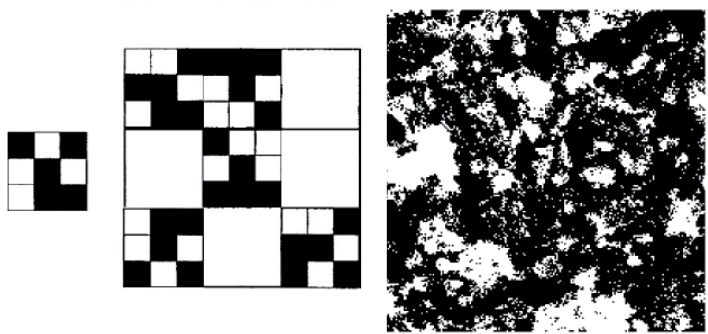

Fig. 1: Statistical self-affinity fractal structure of SEM pictures of robot laser hardened specimens.

Models of fractal lines and surfaces were created to describe the properties of the microstructure of materials. The subject of fractal scan be used to assist in the analysis of surfaces encountered in robot laser hardening. Robot laser hardening [5-8] is a metal surface treatment process complementary to the conventional flame and induction hardening processes. A high-power laser beam is used to heat a metal surface rapidly and selectively to produce hardened case depths of up to $1.5 \mathrm{~mm}$ with hardness values of up to 65 HRc. A hard martensitic microstructure provides improved properties such as wear resistance and increased strength. To harden the work piece, the laser beam usually warms the outer layer to just under the melting temperature. The aim of this study is to see the use of statistical methods in Mechanical Engineering, in order to analyse SEM images of robot laser hardened specimens.

\section{Materials preparation, Methodology and Statistical Method}

First, we hardened tool steel with a robot laser cell. We changed two parameters, speed $\mathrm{v} \in[0,5]$ $\mathrm{mm} / \mathrm{s}$, and temperature $\mathrm{T} \in[850,2000]{ }^{\circ} \mathrm{C}$. After hardening, we polished and etched all specimens. Detailed characterisation of their microstructure before and after surface modifications was conducted using a field emission scanning electron microscope (SEM), JEOL JSM-7600F. The SEM pictures were converted into binary images, from which we calculated the fractal dimension. Fractal dimensions were determined using the Hurst exponent $\mathrm{H}$ estimation method.

We present three methods for calculating the fractal dimension. First, we present the boxcounting model $[9,10]$, to calculate the fractal dimension. Box counting is a method of gathering data for analysing complex patterns by breaking a dataset, object, image, etc. into smaller and smaller pieces, typically "box" shaped, and analysing the pieces at each smaller scale. Theoretically, the intent of box counting is to quantify fractal scaling, but from a practical perspective this would require that the scaling be known ahead of time. To analyse the possibility of the application of fractal analysis to the heat-treated surface, we examined the relation between the surface of mechanical property and fractal dimensions, depending on various parameters of the robot laser cell. In fractal geometry, the key parameter is the fractal dimension $\mathrm{D}$. The relationship between the fractal dimension $\mathrm{D}$, volume $\mathrm{V}$ and length $\mathrm{L}$, can be indicated as follows:

$$
\mathrm{V} \sim \mathrm{L}^{\mathrm{D}} .
$$

Fractal dimensions were determined using the box-counting method, which has been proven to have higher calculation speed and more accuracy by Dougan [11] and Shi [12].

Secondly, we present the homeomorphic regular fractal model $[13,14]$, to calculate the fractal dimension. This method is similar to the boxcounting method. In this case we use a circle or ellipse and not a box/square. Fractal dimensions quantify such fracture models, and the noninteger exponents can be used for estimating the mechanical properties of the fracture surfaces of robot laser hardened specimens. 


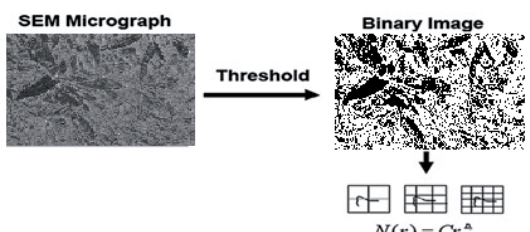

$N(r)=C r^{2}$

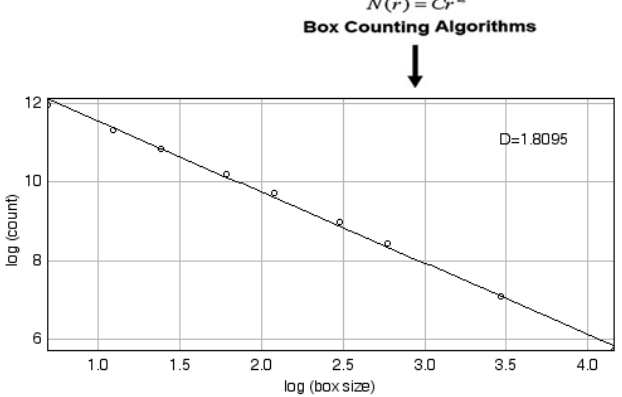

Fig. 2: Calculation of fractal dimensions with box-counting method.
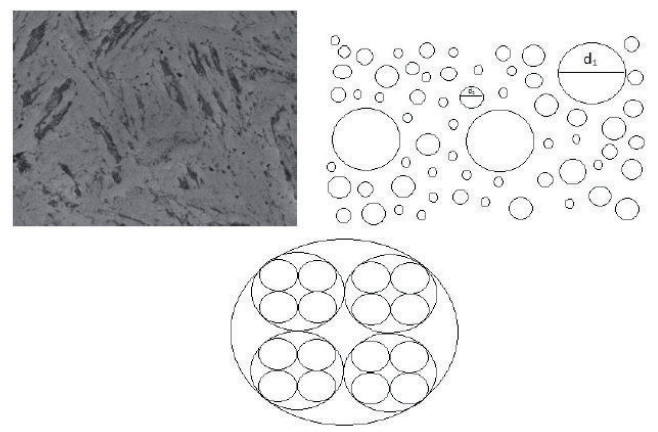

Fig. 3: Calculation of fractal dimensions with homeomorphic model.

Thirdly, we present the R/S method $[15,16]$. The $\mathrm{R} / \mathrm{S}$ method (adjusted rescaled range method) or the adjusted scale is a graphical method and was selected for the estimation of the Hurst exponent. Hurst, the discoverer of the exponent that bears his name, studied power laws as they related to the Nile river floods. The adjusted scale of the partial summation area space component series deviates from the mean. Following Feder, the R/S analysis is as follows. Let the time series of natural phenomena in discrete time in the space component period $\tau$ be $\left\{x_{1}, x_{2}, \ldots, x_{n}\right\}$. The calculation of the average distance $\mathrm{m}$ for the period $\mathrm{t}$ is presented:

$m=\left(x_{1}+x_{2}+\ldots+x_{n}\right) / n$

$Z(\mathrm{t})$ is calculated as in Eq. (2).

$Z(\mathrm{t})=\left(x_{1}-m\right)+\left(x_{2}-m\right)+\ldots+\left(x_{n}-m\right)$

$R(\mathrm{n})$ is calculated as:

$R(\mathrm{n})=\max \left(Z_{1}, Z_{2}, \ldots, Z_{n}\right)-\min \left(Z_{1}, Z_{2}, \ldots, Z_{n}\right)$
$S(\mathrm{n})^{2}$ is calculated as:

$S(\mathrm{n})^{2}=\left(x_{1}-m\right)^{2}+\left(x_{2}-m\right)^{2}+\ldots+\left(x_{n}-m\right)^{2}$

Hurst observed the relationship R/S for a large number of natural phenomena and found the following empirical relationship:

$$
R / S=(c \cdot \tau)^{H} .
$$

The relationship between Hurst's exponent $H$ and the box-counting method for determining the fractal dimension $D$ is very simple. It is presented (in the plane):

$$
D=2-H \text {. }
$$

We use the statistical method of multiple regression $[17,18]$ to predict the mechanical properties of robot laser hardened specimens. Multiple linear regression attempts to model the relationship between two or more explanatory variables and a response variable by fitting a linear equation to observed data. Every value of the independent variable $\mathrm{x}$ is associated with a value of the dependent variable y. Formally, the model for multiple linear regression, given n observations, is

$y_{i}=\beta_{0}+\beta_{1} \cdot x_{i 1}+\beta_{2} \cdot x_{i 2}+\ldots+\beta_{p} \cdot x_{i p}+\beta_{i}$

for $i=1,2, \ldots n$.

In the least-squares model, the best-fitting line for the observed data is calculated by minimising the sum of the squares of the vertical deviations from each data point to the line (if a point lies on the fitted line exactly, then its vertical deviation is $0)$.

\section{Results}

In Table 1, the parameters of hardened specimens that have an impact on hardness are presented. We mark specimens from P1 to P22. Parameter $\mathrm{X}_{1}$ represents the parameter of temperature $\left[{ }^{\circ} \mathrm{C}\right]$, $\mathrm{X}_{2}$ represents the speed of hardening $[\mathrm{mm} / \mathrm{s}], \mathrm{X}_{3}$ represents the fractal dimension calculated with box-counting, $\mathrm{X}_{4}$ represents the fractal dimension calculated with homeomorphic model, $\mathrm{X}_{5}$ represents the fractal dimension calculated with the $\mathrm{R} / \mathrm{S}$ method and $\mathrm{X}_{6}$ represents the percentage of black pixels (before the process of hardening). The final parameter presents the percentage of black pixels of SEM images of robot laser hardened specimens. With the fractal dimension we describe the complexity of hardened specimens. In Table 1, we can see that specimen P11 has the largest fractal dimension, 1.9784, calculated with boxcounting method. Specimen P5 has the largest 
fractal dimension, 1.8065, calculated with the homeomorphic model. Specimen P15 has the largest fractal dimension, 1.456, calculated with the R/S method. Specimen P14 has minimal black pixels of SEM images of robot laser hardened specimens, 19.858\%. Specimen P22 has the maximum percentage of black pixels. Table 2 presents statistical properties of the parameters of robot laser hardened specimens P1-P22. Table 2 presents statistical properties of robot laser hardened specimens P1-P22. In Table 4, the experimental and prediction data are presented. $S$ represents the name of the specimens, ED represents experimental data. Predictions with multiple regression are presented in column PMR. The measured and predicted black pixels of the SEM pictures of robot laser hardened specimens are shown in the graph in Fig. 4. The regression model is presented below. The model of multiple regression PMR1 presents a $14.56 \%$, the model of multiple regression PMR2 $27.46 \%$, the model of multiple regression PMR3 $44.02 \%$ and the model of multiple regression PMR4 6.88 \% deviation from the measured data.

Model of multiple regression PMR1

\section{$Y=-55,1455-0,01409 \times X 1-2,50346 \times X 2+60,4$} $6287 \times X 3+0,122667 \times X 6$

Model of multiple regression PMR2

$$
Y=89,72848-0,00976 \times X 1-
$$

\section{$1,68654 \times X 2+25,1163 \times X 4-0,146841 \times X 6$}

Model of multiple regression PMR3

$$
Y=13,89629-0,01607 \times X 1-
$$

$2,73985 \times X 2+31,95797 \times X 5-0,202305 \times X 6$

Model of multiple regression PMR4

$$
\begin{gathered}
Y=-55,2097-0,00687 \times X 1- \\
1,2046 \times X 2+74,45655 \times X 3- \\
29,7657 \times X 4+7,25088 \times X 5+ \\
0,055399 \times X 6
\end{gathered}
$$

Table 1: Parameters of hardened specimens.

\begin{tabular}{|l|l|l|l|l|l|l|l|}
\hline S & $\mathbf{X 1}$ & $\mathbf{X}$ & $\mathbf{X 3}$ & $\mathbf{X}$ & $\mathbf{X}$ & $\mathbf{X}$ & $\mathbf{Y}$ \\
\hline P1 & 1000 & 2 & 1,9135 & 1,7365 & 1,366 & 84,436 & 38,552 \\
\hline P2 & 1000 & 3 & 1,9595 & 1,6680 & 1,373 & 84,436 & 44,695 \\
\hline P3 & 1000 & 4 & 1,9474 & 1,6871 & 1,366 & 84,436 & 42,660 \\
\hline P4 & 1000 & 5 & 1,9384 & 1,7458 & 1,381 & 84,436 & 40,922 \\
\hline P5 & 1400 & 2 & 1,9225 & 1,8065 & 1,380 & 84,436 & 36,446 \\
\hline P6 & 1400 & 3 & 1,9781 & 1,4956 & 1,382 & 84,436 & 49,225 \\
\hline P7 & 1400 & 4 & 1,954 & 1,1664 & 1,371 & 84,436 & 45,404 \\
\hline P8 & 1400 & 5 & 1,9776 & 1,4784 & 1,356 & 84,436 & 48,245 \\
\hline P9 & 1000 & 2 & 1,972 & 1,6220 & 1,409 & 38,552 & 45,467 \\
\hline P10 & 1000 & 3 & 1,8580 & 1,7426 & 1,362 & 44,695 & 31,727 \\
\hline P11 & 1000 & 4 & 1,9784 & 1,6142 & 1,392 & 42,660 & 45,431 \\
\hline P12 & 1000 & 5 & 1,941 & 1,6982 & 1,355 & 40,922 & 42,309 \\
\hline P13 & 1400 & 2 & 1,9783 & 1,6743 & 1,343 & 36,446 & 36,116 \\
\hline P14 & 1400 & 3 & 1,5810 & 1,3634 & 1,399 & 49,225 & 19,858 \\
\hline P15 & 1400 & 4 & 1,9650 & 1,5155 & 1,456 & 45,404 & 46,150 \\
\hline P16 & 1400 & 5 & 1,8113 & 1,5342 & 1,397 & 48,245 & 35,778 \\
\hline P17 & 800 & 0 & 1,9669 & 1,6528 & 1,375 & 84,436 & 46,516 \\
\hline P18 & 1400 & 0 & 1,9753 & 1,2996 & 1,406 & 84,436 & 52,332 \\
\hline P19 & 2000 & 0 & 1,9706 & 1,6427 & 1,381 & 84,436 & 50,766 \\
\hline P20 & 950 & 0 & 1,9631 & 1,1693 & 1,321 & 84,436 & 68,155 \\
\hline P21 & 850 & 0 & 1,9537 & 1,1042 & 1,381 & 84,436 & 74,245 \\
\hline P22 & 0 & 0 & 1,9721 & 0,7332 & 1,371 & 84,436 & 84,436 \\
\hline
\end{tabular}


Table 2: Statistical properties of parameters of robot laser hardened specimens P1-P22.

\begin{tabular}{|c|c|c|c|c|c|c|c|}
\hline & X1 & $X_{2}$ & X3 & $X 4$ & X5 & $X 6$ & $Y$ \\
\hline Average & 1145 & 2,54 & 1,9308 & 1,506 & 1,3783 & 69,466 & 46,610 \\
\hline Max & 2000 & 5,00 & 1,9784 & 1,8065 & 1,456 & 84,436 & 84,436 \\
\hline Min & 0 & 0,00 & 1,5810 & 0,7332 & 1,321 & 36,446 & 19,858 \\
\hline
\end{tabular}

Table 3: Statistical properties of robot laser hardened specimens P1-P22 for multiple regression model PMR4.

\begin{tabular}{|l|l|}
\hline SS Regression & 3730,576 \\
\hline SS Residual & 394,996 \\
\hline R2 & 0,904257 \\
\hline Std Error of Estimate & 5,131576 \\
\hline F ratio via R2 & 23,61148 \\
\hline
\end{tabular}

Table 4: Experimental and prediction data of robot laser hardened specimens.

\begin{tabular}{|l|l|l|l|l|l|}
\hline S & ED & PMR1 & PMR2 & PMR3 & PMR4 \\
\hline P1 & 38,552 & 50,81079 & 40,93403 & 70,57999 & 40,87794 \\
\hline P2 & 44,695 & 51,08862 & 38,09214 & 69,31021 & 45,18805 \\
\hline P3 & 42,660 & 47,85356 & 36,7095 & 66,18367 & 42,46325 \\
\hline P4 & 40,922 & 44,80594 & 35,24901 & 63,15619 & 38,95005 \\
\hline P5 & 36,446 & 45,71896 & 36,80398 & 64,43961 & 36,81796 \\
\hline P6 & 49,225 & 46,59537 & 33,71344 & 63,48621 & 49,04414 \\
\hline P7 & 45,404 & 42,61662 & 32,63974 & 59,96659 & 55,74191 \\
\hline P8 & 48,245 & 41,54008 & 30,36045 & 57,98095 & 46,89883 \\
\hline P9 & 45,467 & 48,71942 & 32,72707 & 63,16697 & 46,41168 \\
\hline P10 & 31,727 & 40,07673 & 34,80583 & 58,02667 & 33,12882 \\
\hline P11 & 45,431 & 44,60338 & 29,79647 & 58,72287 & 44,81549 \\
\hline P12 & 42,309 & 39,62541 & 28,79407 & 54,43618 & 37,96133 \\
\hline P13 & 36,116 & 43,21204 & 28,35308 & 56,51745 & 41,98823 \\
\hline P14 & 19,858 & 18,2482 & 38,52424 & 43,66275 & 21,56275 \\
\hline P15 & 46,150 & 38,49377 & 26,63196 & 52,42176 & 44,62372 \\
\hline P16 & 35,778 & 27,04567 & 29,22297 & 45,34472 & 31,14812 \\
\hline P17 & 46,516 & 61,86443 & 44,9179 & 80,98025 & 51,19377 \\
\hline P18 & 52,332 & 53,91832 & 38,85092 & 71,60669 & 58,43523 \\
\hline P19 & 50,766 & 45,18014 & 33,11297 & 61,81449 & 43,5694 \\
\hline P20 & 68,155 & 59,52117 & 43,54934 & 78,44831 & 63,8805 \\
\hline P21 & 74,245 & 60,36182 & 44,76143 & 79,7549 & 66,24041 \\
\hline P22 & 84,436 & 73,45084 & 52,59529 & 94,00243 & 84,42048 \\
\hline
\end{tabular}




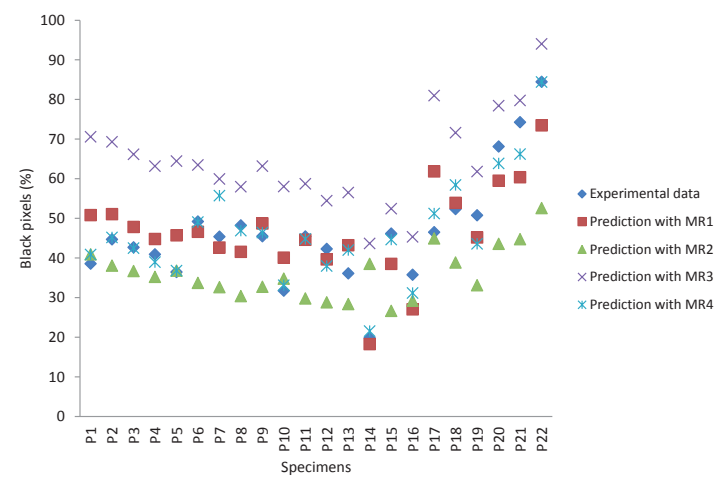

Fig. 4: Measured and predicted black pixels of SEM pictures of robot laser hardened specimens.

\section{Discussion}

Statistical methods are useful in Mechanical Engineering. We used the statistical method of multiple regression to describe the parameters of robot laser cell for hardening, complexity of robot laser hardened specimens and percentage of black pixels of SEM images of robot laser hardened specimens. The fractal approach is more appropriate in the characterisation of complex and irregular surface microstructures observed in the surface of robot laser hardened specimens, and can be effectively utilised for predicting the properties of material from the fractal dimensions of the microstructure. The fractal analysis of a series of digitised surface microstructures from the robot laser surface modified specimens indicated that useful correlations can be derived between the fractal dimensions and the surface microstructural features such as the percentage of black pixels of the SEM images of robot laser hardened specimens. In this paper, we describe how the parameters (viz. speed and temperature) of the robot laser cell affect the percentage of black pixels of the SEM images of robot laser hardened specimens using a new method, fractal geometry. In models of multiple regression PMR1, multiple regression PMR2 and multiple regression PMR3 have the greatest impact on fractal dimension. Models of multiple regression PMR4 have the greatest impact on fractal dimension calculated with the boxcounting method. Statistical properties of robot laser hardened specimens P1-P22 for model multiple regression PMR4 are SS Regression is 3730.576, SS Residual is 394.996, R2 is 0.904257 , standard error of the estimate is 5.131576 and
$F$ ratio via $R 2$ is 23.61148 . The model of multiple regression PMR1 presents a $14.56 \%$, the model of multiple regression PMR2 27.46\%, the model of multiple regression PMR3 $44.02 \%$ and the model of multiple regression PMR 4 6.88\% deviation from the measured data.

\section{Conclusions}

Traditionally, statistics were concerned with drawing inferences using a semi-standardised methodology that was "required learning" in most sciences. In this paper we used a statistical method in Mechanical Engineering. Fractals are a very good method to describe the complexity of patterns. Fractal structures are also found in robot laser-hardened samples when viewed under sufficient magnification. Using the R/S method, the homeomorphic model and the box-counting method, we analysed specimens of equaltempered metal after subjecting them to robot laser hardening using various parameters. The main findings can be summarised as follows.

A fractal structure exists in robot laser hardening. We used three methods: R/S method, homeomerphic model, and box-counting method to calculate the fractal dimensions for different parameters of robot laser cell for hardening. The dependence of the fractal dimension on the percentage of black pixels of the SEM images of robot laser hardened specimens was ascertained. This finding is important if we know that certain alloys mix poorly because they have different melting temperatures, but such alloys have much higher percentage of black pixels of the SEM images of robot laser hardened specimens and better technical characteristics. By varying different parameters (i.e. temperature and speed), robot laser cells produce different fractal patterns with different fractal dimensions. We use the statistical method of multiple regression to analyse robot laser hardened specimens

\section{References}

[1] DeHeer, W. (1999): International response trends: results of an international survey. JOS, 15, 129-142.

[2] B. B. Mandelbrot, The fractal geometry of nature. New York: WH Freeman, 1982, p. 93.

[3] J.M. Barbaroux, F. Germinet, and S. Tcheremchantsev, Fractal dimensions and the phenomenon of intermittency in quantum dynamics, Duke Math. J. 110 (2001), 161-193. 
[4] J.-M. Barbaroux, F. Germinet, and S. Tcheremchantsev, Generalized fractal dimensions: equivalences and basic properties, J. Math. Pures Appl. 80 (2001), 977-1012.

[5] M. Babič. Fractal dimension of the robotically laser hardening tool steel. In: M. Robnik and d. Korošak (eds.), 9th Symposium of Physicists, University of Maribor, Hotel Piramid, Maribor, 9-11 December 2010. Book of Abstracts. Maribor: CAMTP, 2010.

[6] M. Babič, M. Milfelner, S. Stepišnik: Robot laser hardening metals. Y: Perme, Tomaž(ur.), Švetak, Darko (ur.), Balič, Jože (ur.). IRT Industrial Forum, Portoroz, 7 -8. June, 2010. Source of knowledge and experience for the profession: Proceedings of the Forum. Skofljica: Profidtp, 2010. (In Slovene)

[7] Tesar, J., Vacikova, P., Soukup, O., Houdkova, S. (2012). Infrared camera analysis of laser hardening, Advances in Optical Technologies, Vol. 2012, 1-6, doi: 10.1155/2012/593893.

[8] El-Batahgy, A.-M., Ramadan, R.A., Moussa, A.-R. (2013). Laser surface hardening of tool steels - experimental and numerical analysis, Journal of Surface Engineered Materials and Advanced Technology, Vol. 3, No. 2, 146-153, doi: 10.4236/ jsemat.2013.32019.

[9] Nirupam Sarkar and B. B. Chaudhuri: An efficient approach to estimate fractal dimension of textural images, Pattern Recognition, 25, 9, 1035-1041(1992).

[10] S. Appleby: Multifractal characterization of the distribution pattern of the human population, Geographical Analysis 28,147-160(1996).

[11] L.T. Dougan, P.S. Addison, Estimating the cut-off in fractal scaling of fractured concrete, Cement and Concrete Research 31 (2001) 1043-1048.

[12] Y. Shi, X.P. Lou, S.H. Quan, Fractal dimension computation methods for gas diffusion layer of PEM fuel cells (in Chinese), Journal of Wuhan University of Technology 29 (2005) 79-82.

[13] P. Streitenberger, D. Forster, G. Kolbe and P. Veit: The fractal geometry of grain boundaries in deformed and recovered zinc, Scripta Metallurgica et Materiala, 33, 541-546(1995). 9.

[14] L. T. Dougan, P. S. Addison and W. M. C. McKenzie: Fractal analysis of fracture: A comparison of dimension estimates, Mech. Res. Commun, 27, 383-392(2000).

[15] Chun-Feng Li: Rescaled -range and power spectrum analyses on well-logging data, Int. Journal of Geophysics, 153, 201-212(2003)

[16] J. Beran, R. Sherman, M. S. Taqqu, W. Willinger: Long-range dependence in variable-bit-rate video traffic, IEEE T Commun, 43,1566-1579 (1995).

[17] Box, G. E. P. (1954). "Some Theorems on Quadratic Forms Applied in the Study of Analysis of Variance Problems, I. Effect of Inequality of Variance in the One-Way Classification". The Annals of Mathematical Statistics 25 (2): 290. doi:10.1214/aoms/1177728786.

[18] Narula, Subhash C.; Wellington, John F. (1982). "The Minimum Sum of Absolute Errors Regression: A State of the Art Survey". International Statistical Review 50 (3): 317-326. doi:10.2307/1402501. JSTOR 1402501.

\section{Biographical notes}

Matej Babič, born 7th March 1983. He received his Ph.D. degreein Computer Science from the Faculty of Electrical Engineering and Computer Science. He studied Mathematics at the Faculty of Education in Maribor. Current position: Assistant with Ph.D. in Jožef Stefan Institute. He research interests are fractal geometry, graph theory, intelligent systems, hybrid machine learning, and the topography of materials after hardening. 